\title{
Improving transthoracic echocardiography (TTE) training for junior doctors in Harefield Hospital during COVID-19
}

\author{
Authors: Sarah Williamson ${ }^{A}$ and Anthony Barron ${ }^{A}$
}

\section{Introduction}

We devised a medical education quality improvement (QI) project aimed at enhancing training in transthoracic echocardiography (TTE) for junior doctors at Harefield Hospital, Royal Brompton and Harefield NHS Foundation Trust. During the COVID-19 pandemic, junior doctors expressed a desire for further training in the use of transthoracic echocardiography (TTE), which was felt to be particularly helpful in the bedside assessment of fluid status in COVID-19 inpatients. Normally this was achieved through local tutorials. However, there was a moratorium on all faceto-face teaching, including simulation training in non-clinical environments. We attempted to circumvent this by creating an online training module which could be accessed remotely and utilised alongside self-directed study with our local echocardiography simulator.

\section{Materials and methods}

We undertook two plan, do, study, act (PDSA) cycles. PDSA 1 - Plan: A needs assessment of current junior doctors was conducted. Do: Six 20-minute video tutorials were created to form a module. Study: Feedback was obtained from a small focus group of junior doctors. Act: Changes were made to the content and presentation based on their feedback. PDSA 2 - Plan: Online module content, assessment and evaluation were formulated. Do: The TTE module was published online with options for self-study on the local TTE simulator. Study (ongoing): Module evaluations are collected and collated. Act (ongoing): Improvements were/are made to the module based on feedback received.

\section{Results and discussion}

Owing to the small size of our hospital, only a small number of junior doctors have accessed the online module $(n=8)$ since its publication. However, all of these found it useful and easily accessible. Feedback from the focus group in first PDSA cycle demonstrated that junior doctors value accessibility and brevity in online content. Therefore, the module was designed to cover a few short topics, accessible from all devices. It also confirmed that performing needs assessments before designing online learning is crucial, as $8 / 8$ junior doctors accessing the online module felt the content was relevant. The online assessments demonstrated learning with an average post-course assessment score of $78 \%$. Evaluations suggested increased junior doctor knowledge of fluid assessment with TTE and confidence in attempting bedside TTE. Interestingly, although all junior doctors accessing the module intended to, none accessed our echocardiography simulator for self-study despite $5 / 5$ of the focus group suggesting that an instructor was not necessarily essential to learn procedural skills.

\section{Conclusion}

This QI project fulfilled its main aims, as it increased the availability and accessibility of teaching resources in our local hospital. Given the imminent need for alternatives to face-toface training, online modules like this are a sustainable option for procedural training. However, the question of whether they should replace or enhance face-to-face tutelage remains and deserves further investigation. It would also be useful to explore the barriers and enablers for self-directed study on simulators. If adopted, online procedural-training modules offer a variety of options for future training including as an adjunct to skills training in a lab or clinical training on the wards.

\section{Conflicts of interest}

None declared. 